\title{
Regorafenib adjusted dose for Chilean patients with chemoresistant metastatic colorectal cancer: a case series
}

\author{
José Luis Leal ${ }^{1,2}$, Juan Briones ${ }^{3,4}$, María Elisa Herrera ${ }^{5}$, Bettina Müller ${ }^{1}$, Bruno Nervi ${ }^{4}$ and Sebastián Mondaca ${ }^{3,4}$ \\ ${ }^{1}$ Medical Oncology Responsability Centre, Instituto Nacional del Cáncer, Santiago 8380455, Chile \\ ${ }^{2}$ Medical Oncology Service, Instituto Oncológico Fundación Arturo López Pérez, Santiago 7501066, Chile \\ ${ }^{3}$ Hemato-Oncology Department, Complejo Asistencial Sótero del Río, Puente Alto, Santiago 8207257, Chile \\ ${ }^{4}$ Hemato-Oncology Department, Pontificia Universidad Católica de Chile, Santiago 8330032, Chile \\ ${ }^{5}$ Hemato-Oncology Department, Hospital Base de Valdivia, Valdivia 5090146, Chile
}

Correspondence to: José Luis Leal. Email: ㅆlleal@uc.cl

\begin{abstract}
Background: Regorafenib is a therapeutic alternative for patients with metastatic colorectal cancer (MCRC) resistant to conventional therapies. The reported toxicity is relevant and there is no data on Latin American patients. The objective was to evaluate the overall survival (OS), progression-free survival (PFS) and quality of life (QoL) in a prospective cohort of Latin American patients treated with an adjusted dose of regorafenib.
\end{abstract}

Methods: We prospectively recruited patients with MCRC that progressed to standard therapy. A dose escalation algorithm was used. OS, PFS, response rate and QoL were evaluated.

Results: We recruited 13 patients between June and November 2015. The median age was 60 years. Median OS was 8.6 months and median PFS was 2.2 months. The response rate was $8 \%$. Grade $3-4$ toxicities included grade 3 palmoplantar erythrodysesthesia in $23 \%$ and grade 3 fatigue in $12 \%$ of patients.

Conclusion: Regorafenib treatment is effective in Latin American patients with conventional therapy resistant MCRC.

Keywords: regorafenib, antiangiogenic, colorectal neoplasms, Latin America

Published: 02/10/2018

Received: 06/05/2018

ecancer 2018, 12:875 https://doi.org/10.3332/ecancer.2018.875

Copyright: $\odot$ the authors; licensee ecancermedicalscience. This is an Open Access article distributed under the terms of the Creative Commons Attribution License (http://creativecommons.org/licenses/by/3.0), which permits unrestricted use, distribution, and reproduction in any medium, provided the original work is properly cited. 


\section{Background}

Colorectal cancer ( $\mathrm{CRC}$ ) is a common disease, being the second most frequent cancer type in women and the third in men worldwide [1]. In the past decade, we have observed a significant improvement in clinical outcomes associated with the development of new treatment strategies for metastatic CRC, and median survival has been prolonged from 6 months to over 30 months, with the sequential use of conventional chemotherapy (CT) including fluorouracil, irinotecan, oxaliplatin, in combination with monoclonal antibodies such as bevacizumab, cetuximab or panitumumab $[2,3]$.

Many patients maintain a good performance status until progression with or after these therapies and are candidates for other treatments, but options are limited, which is why an unmet need exists. Many trials have examined the role of cytotoxic CT for resistant to standard therapies metastatic CRC patients [4] with discrete results. The RECOURSE trial demonstrated the activity of TAS-102, a combination of triflouridine and tipiracil, compared to placebo, showing benefit in OS [5]. Regorafenib is an orally administered intracellular multi-kinase inhibitor that binds to several intracellular kinases, with a strong effect against vascular endothelial growth factor receptors (VEGFR) 1 to 3, platelet derived growth factor receptor (PDGFRB), fibroblast growth factor receptor 1 (FGFR1) and oncogenic mutant kinases KIT, RET and BRAF [6]. The reported toxicity associated with this drug is relevant, which raises doubts concerning the currently approved dose [7], thus the benefit of an adjusted dose is being evaluated [8].

Regorafenib is a standard treatment option in chemoresistant metastatic CRC based on two phase III trials [9, 10], and is recommended in treatment guidelines [11]. However, the Latin American population has not been represented in either of these studies. Therefore, we cannot rule out the possibility of there being a difference in effectiveness as well as in tolerance to this drug in this population. Interactions have been reported between ethnicities and these variables [12, 13].

The objective of this series was to evaluate the OS, progression-free survival (PFS) and quality of life (QoL) of regorafenib in a cohort of Latin American patients following at least two lines of systemic treatment.

\section{Methods}

\section{Patients}

A prospective cohort was designed, in which patients treated at two hospitals in Chile were enrolled between June and November 2015.

Patients had to be over 18 years, diagnosis of CRC by biopsy, Eastern Cooperative Oncology Group Performance Status (ECOG-PS) 0 or 1 and metastatic measurable disease by RECIST 1.1 [14]. They must have received two or more lines of CT including: 5-fluorouracil and/or capecitabine, oxaliplatin and irinotecan. We excluded patients with an estimated life expectancy below 3 months, and significant cardiac, hepatic or renal dysfunction. All patients signed a consent form that was approved by the institutional ethics committee.

\section{Procedures}

Treatment with regorafenib was administered once daily from days 1 to 21 of each 28-day cycle until disease progression, death, unacceptable toxic effects or withdrawal of consent. The initial dose was of $80 \mathrm{mg}$ and was re-evaluated in 2 weeks. The dose was increased to 120 $\mathrm{mg} /$ day in case of none or mild toxicity. All patients received palliative care at the physician's discretion.

\section{Evaluations}

Tumour response evaluation was performed by computerised axial tomography using RECIST version 1.1 criteria every 8 weeks during treatment. We assessed patient-reported outcomes using the EORTC-QLQ-C30 questionnaire [15], performed at baseline and every 4 weeks before each cycle. Patients were followed-up every 2 weeks during the first 2 cycles, and then every 4 weeks. Adverse events were registered using the NCI CTCAE manual, 4.0 version [16]. 


\section{Statistical analysis}

A descriptive analysis of the population was performed using central tendency and dispersion measures, according to the nature of the variable. Student's $T$-test was used on paired samples to compare continuous variables. Kaplan-Meier method was applied in order to evaluate OS and PFS.

To evaluate QoL, we performed a linear transformation of the EORTC QLQ-C30 questionnaire into a 0-100 score [17]. For a given score, a decrease of more than 10 points without a successive recovery was considered to be significant [18]. GraphPad Prism 7.0 software was used for the statistical analysis.

\section{Results}

\section{Patients}

We enrolled 13 patients in this series during the study period, $6(46.1 \%)$ were male and $7(53.9 \%)$ female. The median age of patients was 60 years (range: $43-76)$ and $8(61 \%)$ had ECOG 1 . The most common primary site was the colon in $77 \%$ of patients. In this cohort, only six patients had a RAS study and of these, two had a gene mutation. Forty-six percent of patients had received more than two lines of CT. There were no patients previously treated with biological therapies. Patient's main characteristics are summarised in Table 1.

\section{Response and survival}

The average dose of regorafenib was of $95.5 \mathrm{mg}$. The average number of cycles was 4.1 (range: 1-9, standard deviation $4.1 \pm$ 2.619) and the median follow-up time was 5.4 months. One patient (8\%) had a partial response and three patients (23\%) had stable disease. All patients were included in the survival analysis. Median PFS and OS were 2.2 and 8.6 months, respectively (Figure 1). Survival at 6 months was $58 \%$. At the time of analysis, 11 patients had suspended treatment due to progression and 1 due to toxicity (grade 3 fatigue).

\section{Toxicity and quality of life}

The most frequent grade 3-4 toxicity was grade 3 palmoplantar erythrodysesthesia (23\%). There was neither grade 4 toxicity nor treatment-related deaths (Table 2). Average weight at the beginning and end of treatment were $70.4 \mathrm{~kg}$ and $65.3(P=0.006)$, respectively. The median time to QoL definitive deterioration was 3 months.

\section{Discussion}

Our series suggests that regorafenib is effective and tolerable in Latin American patients with metastatic CRC (MCRC) previously treated with cytotoxic CT. In this study, regorafenib showed a discrete activity and an acceptable toxicity, which is consistent with previous results $[9,10]$. The CORRECT study included patients with resistant metastatic CRC that had received all standard therapies. In the group treated with regorafenib, the median OS was 6.4 months and the PFS was 1.9 months; the objective response rate was $10 \%$. In our series, the OS was slightly better, reaching a median of 8.6 months. This may be explained because, unlike CORRECT, our patients had not received any previous biological therapies including antiangiogenics, which is one of the action mechanisms of regorafenib, or epithelial growth factor (EGFR) inhibitors in cases with wild-type RAS. In Chile, as in other countries with limited resources, these drugs are not covered by the public healthcare system. In CONCUR study, strictly an Asian population, $40 \%$ of patients had not received previous biological treatment and OS and PFS were 8.8 and 3.2 months, respectively. 
Table 1. Basal characteristics of the study population.

\begin{tabular}{|l|c|}
\hline \multicolumn{1}{|c|}{ Characteristic } & Number (\%) \\
\hline Median age (range, year) & $60(43-76)$ \\
\hline Gender & \\
Male & $6(46)$ \\
Female & $7(54)$ \\
\hline ECOG PS & $5(38)$ \\
0 & $8(61)$ \\
1 & \\
\hline Primary Site & $10(77)$ \\
Colon & $3(23)$ \\
Rectum & $11(85)$ \\
\hline Histology & $1(7,5)$ \\
Adenocarcinoma & $1(7,5)$ \\
Mucinous & \\
Other & $4(30)$ \\
\hline RAS Mutation & $2(15)$ \\
No & $7(55)$ \\
Yes & \\
Unknown & $3(23)$ \\
\hline Stage at Debut & $10(77)$ \\
I-III & \\
IV & $7(54)$ \\
\hline Previous CT number & $6(46)$ \\
2 & \\
$>2$ & \\
\hline Previous use of biological & $(100)$ \\
therapy & \\
Yes & \\
No & \\
\hline
\end{tabular}

CT: Chemotherapy

Our data showed low-grade 3-4 toxicity: grade 3 hand-foot syndrome (23\%) and grade 3 fatigue (15\%). This may be due to the protocol we used, in which the maximum standard dose of $160 \mathrm{mg} /$ day was not reached. In the CORRECT study, $58 \%$ of patients developed grade 3 or 4 toxicity and $38 \%$ needed dose reduction [9]. In one phase III-B study, $54 \%$ of patients reported grade 3 adverse events [19].

QoL results in our cohort are concordant with the analysis performed by the pivotal studies, in which there is no evidence of QoL degradation. The plateau in the QoL domain curve represents the subgroup that maintains the treatment due to a clinical benefit. The main limitation of this series is its low number of patients, which precludes the obtainment of precise results. However, we consider that our experience adds new knowledge of the effectiveness and toxicity of regorafenib in Latin American population. We also used a lower dose regimen, which could aid in the issue of cost-effectiveness [20] that is of particular importance for developing countries. In a previous report from a low-resource setting, patients with chemoresistant MCRC received standard dose regorafenib in a cancer centre in India. This study showed similar outcomes in terms of efficacy and toxicity than pivotal phase III trials [21]. In our series, the toxicity seemed lower while maintaining similar efficacy, suggesting that a dose escalation strategy might be a reasonable alternative. 
Overall Survival

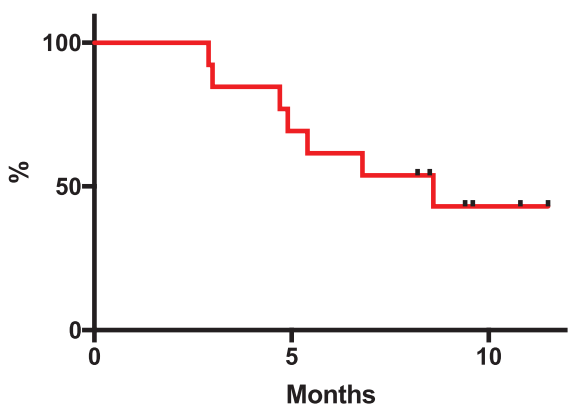

Progression Free Survival

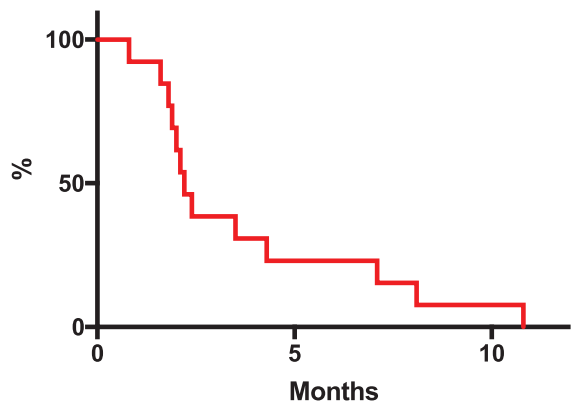

Figure 1. Kaplan Meier plot of OS and PFS.

Table 2. Treatment-related adverse events of the study population.

\begin{tabular}{|l|c|c|}
\hline \multicolumn{1}{|c|}{ Toxicity type } & Grade 3 N (\%) & Grade 4 N (\% \\
\hline Hematologic & - & - \\
\hline Diarrhea & - & - \\
\hline Palmoplantar erythrodysesthesia & $3(23 \%)$ & - \\
\hline Cardiovascular (hypertension) & $1(7 \%)$ & - \\
\hline Fatigue & $2(15 \%)$ & - \\
\hline
\end{tabular}

\section{Conclusions}

Our data suggest that Regorafenib is an effective treatment for Latin American patients with chemoresistant MCRC, with an acceptable toxicity using an adaptive dose escalation schedule. This must be confirmed in larger analytical studies, but might represent a more attractive alternative in terms of safety and cost-effectiveness.

\section{Funding statement}

This research did not receive any specific grant from funding agencies in the public, commercial or not-for-profit sectors. 


\section{Conflicts of interest}

The authors have no conflicts of interest to declare.

\section{References}

1. Ferlay J, Shin HR, and Bray F, et al (2010) Estimates of worldwide burden of cancer in 2008: GLOBOCAN 2008 Int J Cancer 127(12) 2893-2917 https://doi.org/10.1002/ijc.25516

2. Heinemann V, von Weikersthal LF, and Decker T, et al (2014) FOLFIRI plus cetuximab versus FOLFIRI plus bevacizumab as firstline treatment for patients with metastatic colorectal cancer (FIRE-3): a randomised, open-label, phase 3 trial Lancet Oncol 15(10) 1065-1075 https://doi.org/10.1016/S1470-2045(14)70330-4 PMID: 25088940

3. Douillard JY, Oliner KS, and Siena S, et al (2013) Panitumumab-FOLFOX4 treatment and RAS mutations in colorectal cancer N Engl J Med 369(11) 1023-1034 https://doi.org/10.1056/NEJMoa1305275 PMID: 24024839

4. Kang EJ, Choi YJ, and Kim JS, et al (2010) Mitomycin-C, 5-fluorouracil, and leucovorin as a salvage therapy in patients with metastatic colorectal adenocarcinoma Asia Pac J Clin Oncol 6(4) 286-291 https://doi.org/10.1111/j.1743-7563.2010.01334.x PMID: 21114778

5. Mayer RJ, Van Cutsem E, and Falcone A, et al (2015) Randomized trial of TAS-102 for refractory metastatic colorectal cancer N Engl J Med 372(20) 1909-1919 https://doi.org/10.1056/NEJMoa1414325 PMID: 25970050

6. Wilhelm SM, Dumas J, and Adnane L, et al (2011) Regorafenib (BAY 73-4506): a new oral multikinase inhibitor of angiogenic, stromal and oncogenic receptor tyrosine kinases with potent preclinical antitumor activity Int J Cancer 129(1) 245-255 https:// doi.org/10.1002/ijc. 25864

7. Tabchi S and Ghosn M (2015) Regorafenib: start low and go slow Target Oncol 10(3) 445-447 https://doi.org/10.1007/s11523-014$\underline{0352-7}$

8. Bekaii-Saab TS, Ou F-S, and Anderson DM, et al (2018) Regorafenib dose optimization study (ReDOS): randomized phase II trial to evaluate dosing strategies for regorafenib in refractory metastatic colorectal cancer (mCRC) An ACCRU Network study J Clin Oncol 36(4_suppl) 611

9. Grothey A, Van Cutsem E, and Sobrero A, et al (2013) Regorafenib monotherapy for previously treated metastatic colorectal cancer (CORRECT): an international, multicentre, randomised, placebo-controlled, phase 3 trial Lancet 381(9863) 303-312 https://doi.org/10.1016/S0140-6736(12)61900-X

10. Li J, Qin S, and Xu R, et al (2015) Regorafenib plus best supportive care versus placebo plus best supportive care in Asian patients with previously treated metastatic colorectal cancer (CONCUR): a randomised, double-blind, placebo-controlled, phase 3 trial Lancet Oncol 16(6) 619-629 https://doi.org/10.1016/S1470-2045(15)70156-7 PMID: 25981818

11. Schmoll HJ, Van Cutsem E, and Stein A, et al (2012) ESMO Consensus Guidelines for management of patients with colon and rectal cancer. a personalized approach to clinical decision making Ann Oncol 23(10) 2479-2516 https://doi.org/10.1093/annonc/ mds236 PMID: 23012255

12. Yano R, Konno A, and Watanabe K, et al (2013) Pharmacoethnicity of docetaxel-induced severe neutropenia: integrated analysis of published phase II and III trials Int J Clin Oncol 18(1) 96-104 https://doi.org/10.1007/s10147-011-0349-5

13. O'Donnell PH and Dolan ME (2009) Cancer pharmacoethnicity: ethnic differences in susceptibility to the effects of chemotherapy Clin Cancer Res 15(15) 4806-4814 https://doi.org/10.1158/1078-0432.CCR-09-0344 
14. Eisenhauer EA, Therasse P, and Bogaerts J, et al (2009) New response evaluation criteria in solid tumours: revised RECIST guideline (version 1.1) Eur J Cancer 45(2) 228-247 https://doi.org/10.1016/j.ejca.2008.10.026

15. Aaronson NK, Ahmedzai S, and Bergman B, et al (1993) The European Organization for Research and Treatment of Cancer QLQC30: a quality-of-life instrument for use in international clinical trials in oncology J Natl Cancer Inst 85(5) 365-376 https://doi. org/10.1093/jnci/85.5.365 PMID: $\underline{8433390}$

16. National Cancer Institute (2010) Common Terminology Criteria for Adverse Events v4.0 [https://evs.nci.nih.gov/ftp1/CTCAE/ CTCAE 4.03/CTCAE 4.03 2010-06-14 QuickReference 5x7.pdf]

17. Fayers P, Aaronson NK, and Bjordal K, et al (2001) EORTC QLQ-C30 Scoring Manual 3rd edn (Brussels, Belgium: European Organisation for Research and Treatment of Cancer)

18. Satoh T, Bang YJ, and Gotovkin EA, et al (2014) Quality of life in the trastuzumab for gastric cancer trial Oncologist 19(7) 712-719 https://doi.org/10.1634/theoncologist.2014-0058 PMID: 24951609 PMCID: 4077451

19. Cutsem EV, Ciardiello F, and Ychou M, et al (2016) Regorafenib in previously treated metastatic colorectal cancer (mCRC): analysis of age subgroups in the open-label phase IIlb CONSIGN trial J Clin Oncol 34(15_suppl) 3524 https://doi.org/10.1200/ JCO.2016.34.15 suppl.3524

20. Deger C, Telli F, and Gunaldi M, et al (2015) The cost-effectiveness of regorafenib in the treatment of patients with metastatic colorectal cancer (Mcrc) who have progressed after standard therapies in Turkey Value Health 18(7) A458 https://doi. org/10.1016/j.jval.2015.09.1176 PMID: 26532572

21. Zanwar S, Ostwal V, and Gupta S, et al (2016) Toxicity and early outcomes of regorafenib in multiply pre-treated metastatic colorectal adenocarcinoma-experience from a tertiary cancer centre in India Ann Transl Med 4(4) 74 PMID: 27004221 PMCID: 4779767 